\title{
Autogenous regulation in vivo of the rpmE gene encoding ribosomal protein L31 (bL31), a key component of the protein-protein intersubunit bridge B1b
}

\author{
LEONID V. ASEEV, LUDMILA S. KOLEDINSKAYA, and IRINA V. BONI \\ Shemyakin-Ovchinnikov Institute of Bioorganic Chemistry RAS, 117997 Moscow, Russia
}

\begin{abstract}
Bacterial ribosomal proteins ( $r$-proteins) encoded by nonessential genes often carry out very important tasks in translation. In particular, this is the case of a small basic bacteria-specific r-protein L31 (bL31). Recent studies revealed a crucial role of bL31 in formation of the protein-protein intersubunit bridge B1b and hence its contribution to ribosome dynamics. Our goal was to study in vivo regulation of the rpmE operon encoding bL31. We used a previously developed approach based on chromosomally integrated fusions with the lacZ reporter. E. coli rpmE is transcribed from two promoter regions, and translation of both mRNA transcripts was shown to be feedback regulated by bL31, indicating that the autogenous operator is located within the shorter transcript. The bL31-mediated control of rpmE is gene-specific, as no regulation was found for rpmE-unrelated reporters. Thus, bL31, as many other r-proteins, possesses dual activity in living cells, acting both as an integral ribosome component and an autogenous repressor. Phylogenetic studies revealed the presence of a highly conserved stem-loop structure in the rpmE 5'UTR, a presumable translational operator targeted by bL31, which was further confirmed by site-directed mutagenesis. This stable operator stem-loop separates an AU-rich translational enhancer from a Shine-Dalgarno element, which is a rare case of a noncontiguous translation initiation region. Sequence/ structure computational approaches classify bL31 as an RNA-binding protein, consistent with its repressor function discovered here. Mutational analysis of bL31 showed that its unstructured amino-terminal part enriched in lysine is necessary for the repressor activity.
\end{abstract}

Keywords: gene expression; autogenous regulation; ribosomal proteins; bL31; YkgM; intersubunit bridge B1b; phylogenetic conservation

\section{INTRODUCTION}

A bacteria-specific ribosomal protein L31 (bL31 according to Ban et al. 2014) is a small (70 amino acid residues in length), basic (isoelectric point 9.87) component of the large ribosomal subunit, which is loosely associated with the ribosome (Eistetter et al. 1999). Although the rpmE gene encoding bL31 is not essential and the corresponding knockout strain exists (Baba et al. 2006), its product carries out very important functions in living cells as a key component of the intersubunit bridge $\mathrm{B} 1 \mathrm{~b}$, the only one formed exclusively by r-proteins (Liu and Fredrick 2016). Protein bL31 contributes to ribosomal subunit association by interacting with uL5 in a central protuberance of the $50 \mathrm{~S}$ subunit via its amino-terminal domain, and with uS13 in a head of the 305 subunit via its carboxy-terminal part (Fischer et al. 2015). Interaction of the carboxy-terminal

Corresponding author: irina_boni@ibch.ru

Article is online at http://www.rnajournal.org/cgi/doi/10.1261/rna. 074237.119. Freely available online through the RNA Open Access option. domain of bL31 with a hydrophobic surface formed by proteins uS14 and uS19, as well as electrostatic interaction between bL31 Arg63 and the phosphate backbone of the 16S rRNA helix h42 (A1311 and G1312) have also been identified, and these interactions were proposed to be referred to as a bridge B1c (Liu and Fredrick 2016). Employing bL31 as a key connecting link, B1b and B1c play a crucial role in ribosome dynamics by helping to cope with ribosome structural flexibility due to obligatory rotational movements of the subunits during the translation process (Shasmal et al. 2010; Fischer et al. 2015; Liu and Fredrick 2016; Chadani et al. 2017; Ueta et al. 2017). In addition, bL31 antagonizes intrinsic ribosome destabilization caused by certain amino acid sequences of the nascent peptide in the exit tunnel (Chadani et al. 2017). Recently published data also highlight the role of bL31 in

(C) 2020 Aseev et al. This article, published in RNA, is available under a Creative Commons License (Attribution-NonCommercial 4.0 International), as described at http://creativecommons.org/licenses/ by-nc/4.0/. 
the initiation of translation and maintaining the reading frame (Lilleorg et al. 2017).

Ribosomal proteins in bacteria are highly conserved, and for the most part each r-protein is encoded by a single gene. However, comparative genomic studies have revealed exceptions to this trend, showing that some r-proteins have paralogs which differ in their ability to bind zinc-ions (Makarova et al. 2001; Panina et al. 2003). The E. coli genome encodes two Zn-binding r-proteins, bL31 and bL36, which have paralogs, $\mathrm{YkgM}$ and $\mathrm{YkgO}$, respectively, lacking the zinc-binding motifs (Hensley et al. 2012). The $y \mathrm{kgM}-y \mathrm{kgO}$ operon is transcriptionally repressed by Zur (zinc uptake regulator), so that under normal zinc supply it is silent (Sigdel et al. 2006; Hemm et al. 2010; Gilston et al. 2014 and below). It is believed that $y \mathrm{kgM}-\mathrm{kkgO}$ is expressed only during zinc-starvation, and its products can replace bL31 and bL36 on the ribosome, thus allowing the cell to use the displaced proteins as a reservoir for Zn-ions essential for many enzymatic activities (Hemm et al. 2010; Hensley et al. 2012). Replacement of bL31 by its paralog YtiA has been reported for Bacillus subtilis ribosomes (Akanuma et al. 2006), and very recently, the replacement of bL31 and bL36 by their paralogs YkgM and $Y \mathrm{kgO}$, respectively, was demonstrated for E. coli in the stationary phase of growth (Lilleorg et al. 2019).

There is a paucity of information concerning the regulation of synthesis of bL31 and bL36 themselves. As to bL36, it is encoded by rpmJ at the very end of the long spc operon which is under the control of the r-protein S8, but the question as to whether rpmJ and the preceding gene secY (encoding a nonribosomal protein) can be regulated by S8 is not yet solved. It seems easier to explore the regulation of the bL31 synthesis. According to available data of differential RNA sequencing (dRNA-seq, see Thomason et al. 2015, E. Hajnsdorf personal communication), the monocistronic operon rpmE of $E$. coli is expressed from two promoter regions. It is likely that the promoter region 1 comprises overlapping promoters, since two closely spaced transcriptional start sites (TSSs) were indicated by both above dRNA-seq arrays. It is also possible that one of these promoters is activated during heat shock, given that bL31 is the only known heat shock-inducible ribosomal protein (Wade et al. 2006; Rasouly and Ron 2009). Overall dRNA-seq data report the presence of three TSSs for E. coli rpmE-two of them correspond to the promoter region 1, and the third TSS -to the promoter P2 (Fig. 1A, see below).

The main goal of this work was to study whether the synthesis of bL31 could be regulated by the mechanism of autogenous control. Regulatory circuits linking synthesis of three rRNAs and dozens of r-proteins have not yet been studied in full even for the best-studied model bacterium E. coli. Many r-protein operons were found to be feedback regulated by their products (Fu et al. 2013 and references therein; Aseev et al. 2015, 2016; Babina et al.

\section{A}

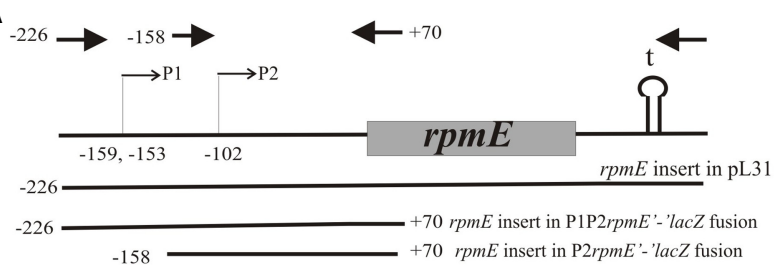

-230 CGGGCAAGG AACGTGGGCA ACGgGCATAg CATCATCCTG ACtTGAAATT CGgTGGGTTA -170 GTATACACAT TGCCGTAGA GATGCGGA CAGTTTGCAT ACGCTGGTTA ATTTCTGTAT $\begin{aligned}-110 & \text { GATTTCGCGC CTTCGTACGA AATGATCGTA TTGAAGCTAT ACTTTTAACA TCGCGTGGTG } \\ -50 & \text { TCTGGCGTTA GGGCTGGAAG AGCGACGCGG CCTTAAACQG AGGTTTCCC ATGAAAAAAG }\end{aligned}$ +11 ATATTCACCC GAAATACGAA GAAATTACTG CTAGCTGCTC TTGCGGTAAC GTAATGAAAA +71 TCCGCTCCAC CGTTGGTCAT GACCTGAACC TCGACGTGTG CAGCAAGTGC CACCCGTTCT +131 TCACTGGCAA ACAGCGTGAT GTTGCTACCG GTGGCCGTGT TGACCGCTTC AACAAGCGTT +191 TCAACATCCC GGGCAGCAAA
+251 TTAGGACACGGA TTTCCGAAAA AAGCGCCGCA CGGCGCTTTT

B

B

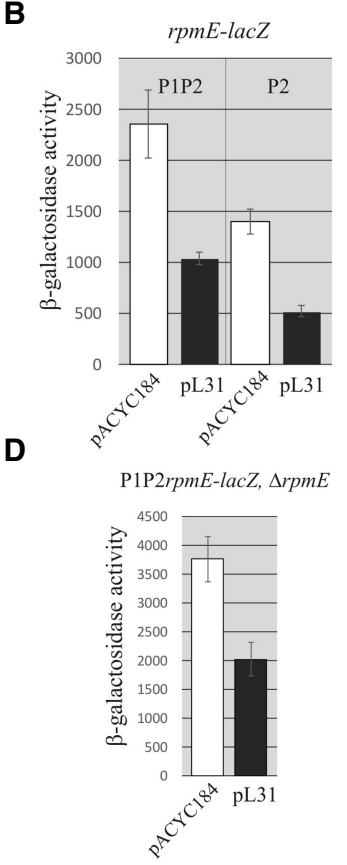

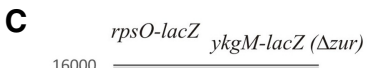

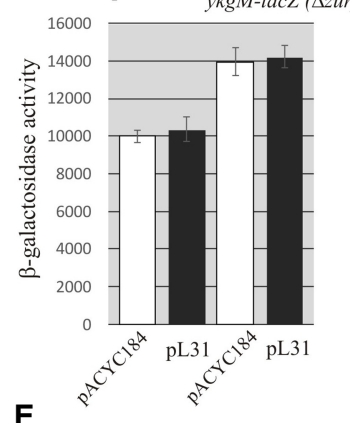

P1P2rpmE-lacZ, $\Delta r p m E$

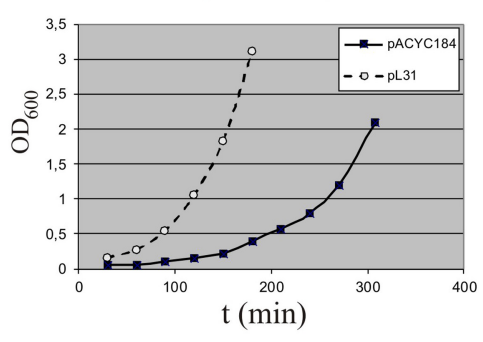

FIGURE 1. Regulation of the rpmE gene encoding bL31. (A) Structure of the rpmE operon; (P1, P2)-promoters, ( $t$ )-terminator. Regions amplified for constructing the plasmid pL31, and P1P2rpmE'-'lacZ and P2rpmE'-'lacZ fusions are designated by lines, with corresponding primers used for PCR indicated by arrows above the operon structure. Below-the sequence of rpmE with flanking regions; start and stop codons as well as an SD-sequence are boxed, transcription start sites are encircled, the $\rho$-independent terminator is underlined. (B) Inhibitory effect of bL31 in trans on the rpmE-lacZ expression under the control of both entire promoter region (P1P2) and P2 alone. The averages of at least 4 independent experiments are represented. The error bars denote standard deviations. $(C)$ The absence of the bL31 effect on the rpmE-unrelated reporters rpsO-lacZ and ykgMlacZ (the latter in a $\Delta$ zur background). (D) Increased expression of P1P2rpmE-lacZ in a bL31-deficient strain. (E) Complementation of the slow-growth phenotype of the $\triangle r p m E$ strain in the presence of pL31.

2015). However, it appeared that some of the operons do not use this mechanism to control their expression (Aseev et al. 2016). The rpmE gene has never been studied, and it was impossible to predict in advance whether it could be regulated by its product or not. By using the 
rpmE'-lacZ chromosomally integrated reporters, we show here for the first time that bL31 in trans is able to modulate its own synthesis in a gene-specific manner. We found the operator site within the mRNA transcript from the promoter P2. Phylogenetic analysis of the 5'-untranslated regions ( $5^{\prime}$ UTRs) of the rpmE genes from $\gamma$ proteobacteria revealed the presence of a highly conserved stem-loop structure bearing specific features, which is typical of the autogenously regulated r-protein operons (Fu et al. 2013; Meyer 2017, 2018; Mustoe et al. 2018). Site-directed mutagenesis provided evidence that the conserved features of the stem-loop, such as two nearly situated bulges, are necessary for the bL31-mediated autogenous control. On the other part, mutational analysis of the rpmE gene within a plasmid producing bL31 in trans revealed that the amino-terminal intrinsically disordered region (residues 2 to 8 ) enriched in lysine is absolutely required for the repressor activity. One more feedback loop found here broadens our knowledge of mechanisms regulating ribosome biogenesis in bacteria.

\section{RESULTS AND DISCUSSION}

\section{Autogenous regulation of the rpmE expression}

Biogenesis of ribosomes is energetically costly and therefore requires multiple control mechanisms to adjust the levels of ribosomal components to cellular demands under various environmental conditions. One of the important mechanisms ensuring coordinated and balanced synthesis of rRNAs and r-proteins is the feedback regulation of r-protein synthesis (Nomura et al. 1984; Zengel and Lindahl 1994). Although at present most of r-protein operons in E. coli (16 out of 21, see Fu et al. 2013; Aseev et al. 2015, 2016; Babina et al. 2015) have been already explored (much less in other bacteria), several operons are still awaiting their turn. Among the latter, there is a monocistronic operon rpmE encoding a small basic r-protein bL31, a key component of the protein-protein intersubunit bridge B1b (see Introduction). Our main goal was to study regulation of the rpmE expression in vivo, which has never been addressed.

According to Escherichia coli dRNA-seq data, the rpmE gene is expressed from two promoter regions (Fig. 1A). Two closely spaced transcription start sites (TSSs) correspond to the promoter region 1, implying the overlapping of two promoters in this region, and one TSS pertains to the downstream promoter P2 (Thomason et al. 2015, E. Hajnsdorf personal communication). To study the rpmE regulation, we generated two reporter constructs and integrated them into the lac region of the chromosome of a specialized ENSO strain (Dreyfus 1988). In the first fusion, expression of the chromosomal lacZ gene was governed by all the natural rpmE promoters and corresponding 5'-UTRs, while in the second one-by the promoter P2 alone and its 5'-UTR (Fig. 1A). The resulting recombinant strains (LAB_P1P2rpmE::lacZ and $L A B_{-} P 2 r p m E::(a c Z)$ were then transformed by the PACYC184-derivative expressing rpmE under the control of its own promoter and terminator regions (further referred to as pL31) and by an empty vector as a control. The $\beta$-galactosidase assay showed a decrease in the lac $Z$ expression caused by $\mathrm{pL} 31$, indicating the inhibitory effect of bL31 in trans on both reporters (Fig. 1B). This effect is 5 '-UTR-specific as no changes in expression was observed for the rpmE-unrelated reporters rpsO-lacZ and ykgM-lacZ (Fig. 1C). It should be mentioned that the expression level of $y \mathrm{kgM}-\mathrm{lac} Z$ corresponding to the rpmE paralogous gene $y \mathrm{kgM}$ was measured in a strain deficient in transcription regulator Zur that completely repressed transcription from the $y \mathrm{kgM}$ promoter under normal conditions (see below). The results allow us to conclude that bL31 acts as a specific repressor of its own synthesis.

It has been observed that the expression of the r-protein operon can be modulated by its product not only when the latter is overexpressed from a plasmid but also under its normal intracellular supply. For instance, these are the cases of rpsA (Boni et al. 2000), rpsB (Aseev et al. 2008), and rpsO (Mathy et al. 2004) autogenous regulators. An intrinsic autogenous repression was found to be alleviated in mutant strains which produced the reduced amount of essential r-proteins S1 or S2 (Boni et al. 2000; Aseev et al. 2008) or no r-protein-repressor at all, as in the case of the S15-null mutant lacking the nonessential gene rpsO (Mathy et al. 2004). The lessening of the intrinsic feedback regulation was observed as an augmented expression of the rpsA-lacZ, rpsB-lacZ, and rpsO-lacZ reporters in corresponding mutant strains. To study whether a similar effect is also typical of the rpmE regulation by bL31, we created the rpmE-deletion mutant bearing the rpmE-lacZ fusion in the chromosomal lac region. The $\beta$-galactosidase assay revealed an elevated expression level of the reporter gene in this strain as compared to the wild-type rpmE background (Fig. 1D). This implies that the rpmE expression is modulated by bL31 even under normal supply of the r-protein during the steady state growth.

\section{Deletion of the chromosomal rpmE gene impairs cell growth}

The rpmE::kan allele was P1 transduced from the JW3907 knockout strain (Baba et al. 2006) into LAB_P1P2rpmE:: lacZ (Table 1). The resulting bL31-deficient cells showed a significant growth defect that was completely suppressed in the presence of pL31 (Fig. 1E). The slow-growth phenotype of the $\triangle \mathrm{rpmE}$ cells is in accordance with the data of Ueta et al. (2017) but contradicts the observation of Lilleorg et al. (2017) that a single deletion of the rpmE gene did not affect the growth of E. coli, and only a 
TABLE 1. Strains and plasmids used in this study

\begin{tabular}{|c|c|c|}
\hline Strain/plasmid & Relevant characteristics & Reference or source \\
\hline $\mathrm{DH} 5 \alpha$ & Cloning host & Laboratory stock \\
\hline ENSO & his, former HfrG6 $\Delta 12$, Lac ${ }^{-}$ & Dreyfus (1988) \\
\hline LAB_P1P2rpmE::lacZ & ENSO bearing chromosomal rpmE'-'lacZ fusion under rpmE promoters & This work \\
\hline LAB_P2rpmE::lacZ & ENSO bearing rpmE'-'lacZ under rpmE promoter P2 & This work \\
\hline LAB_P1P2rpmE::lacZ(GtoC) & LAB_P1P2rpmE::lacZ bearing " $\mathrm{G}$ to $\mathrm{C}$ " mutation in bL31 operator site & This work \\
\hline LAB_P2rpmE::lacZ(GtoC) & LAB_P2rpmE::lacZ bearing " $G$ to $C$ " mutation in bL31 operator site & This work \\
\hline LAB_P1P2rpmE::lacZ( $\Delta$ B2) & LAB_P1P2rpmE::lacZ bearing deletion of AGA in bL31 operator site & This work \\
\hline LAB_P2rpmE::lacZ( $\triangle$ B2) & LAB_P2rpmE::lacZ bearing deletion of AGA in bL31 operator site & This work \\
\hline LAB_P1P2rpmE::lacZ( $\Delta$ ss) & $\begin{array}{l}\text { LAB_P1P2rpmE::lacZ bearing deletion of } 9 \text { bp-region preceding the operator } \\
\text { stem-loop }\end{array}$ & This work \\
\hline $\begin{array}{l}\text { LAB_P1P2rpmE::lacZ( } \Delta B 2 \text {, } \\
\text { sss) }\end{array}$ & Double mutant of LAB_P1P2rpmE::lacZ & This work \\
\hline LAB_ykgM::lacZ & ENSO bearing ykgM'-'lacZ fusion under ykgM promoter & This work \\
\hline LAB_rpmE::lacZ(Y.p) & ENSO bearing rpmE'-'lacZ where rpmE moiety was amplified from Y. pestis DNA & This work \\
\hline IBrpsO188::lacZ & ENSO rpsO'-'lacZ under rpsO promoter & Le Derout et al. (2010) \\
\hline JW3907 & BW25113 $\Delta$ rpmE::kan, Keio collection & Baba et al. (2006) \\
\hline JW5714 & BW25113 $\Delta$ zur::kan, Keio collection & Baba et al. (2006) \\
\hline \multicolumn{3}{|l|}{ Plasmids } \\
\hline $\mathrm{pEMBL} \triangle 46$ & pEMBL8 ${ }^{+}$derivative lacking lacZ RBS & Dreyfus (1988) \\
\hline pEP1P2rpmE-lacZ & $\mathrm{pEMBL} \triangle 46$ bearing $r p m E^{\prime}-{ }^{\prime} l a c Z$ reporter under rpmE promoters $\mathrm{P} 1 \mathrm{P} 2$ & This work \\
\hline pEP2rpmE-lacZ & $\mathrm{pEMBL} \triangle 46$ bearing rpmE'-'lacZ reporter under rpmE promoter P2 & This work \\
\hline pEykgM-lacZ & $\mathrm{pEMBL} \triangle 46$ bearing $y k g M^{\prime}-{ }^{\prime} l a c Z$ reporter under $y k g M$ promoter & This work \\
\hline pACYC184 & tet $^{r}, \mathrm{Cm}^{r}$, cloning vector & Laboratory stock \\
\hline pL31 & pACYC184 derivative expressing rpmE under its entire nature promoter region & This work \\
\hline pL31mut & A set of plasmids bearing mutations in the rpmE coding region & This work \\
\hline
\end{tabular}

simultaneous deletion of the genes encoding the two paralogs, bL31 and YkgM, resulted in a cell-growth defect. The necessity for deleting both paralogous genes to obtain a slow-growth phenotype seems irrational, given that under normal zinc supply the $y k g M-y k g O$ operon should be silent due to $\mathrm{Zn}^{2+}$-dependent repression by a transcriptional regulator Zur (zinc uptake regulator) (Hemm et al. 2010; Gilston et al. 2014). One of the explanations for the inconsistency of the results may be that under growth conditions used by Lilleorg et al. (2017), the $y k g M-y k g O$ operon was partly open, so that YkgM could compensate for the absence of bL31.

To obtain independent data for the extent of transcriptional repression of $y k g M-y k g O$ by Zur, we constructed the $y \mathrm{kgM}$-lacZ chromosomal reporter driven by the $y \mathrm{kgM}$ promoter including the Zur-binding site (Fig. 2A). The cells were grown to exponential phase $\left(\mathrm{OD}_{600} \sim 0.4-0.5\right)$, harvested by centrifugation, and clarified lysates were prepared. The $\beta$-galactosidase assay showed a negligible activity of the reporter, but after transducing the zur::kan al-

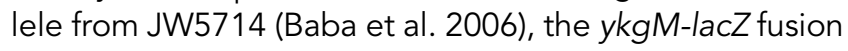
revealed a very high expression level (Fig. 1C). The impressive increase in expression (almost 1000-fold!) was also ob- tained after the treatment of the $z u r^{+}$-cells harboring the ykgM-lacZ reporter with a $\mathrm{Zn}^{2+}$-chelator TPEN (Fig. 2B). These results are fully consistent with previous observations that synthesis of $\mathrm{YkgO}$, the paralog of bL36 and the product of the second gene of the $y \mathrm{kgM}-\mathrm{ykgO}$ operon, was greatly increased after treatment of cells with EDTA as a chelator of bivalent metal ions including $\mathrm{Zn}^{2+}(\mathrm{Hemm}$ et al. 2010). Thus, expression of the $y k g M-y k g O$ operon in a cell can be stimulated either by $\mathrm{Zn}^{2+}$-deficiency or by malfunctioning of the gene encoding Zur-repressor.

To address the question whether YkgM as a paralog of bL31 could modulate the rpmE-lacZ expression, we used a strain deficient in bL31 ( $\Delta$ rpmE) and bearing the P1P2rpmE-lacZ fusion in the chromosome. As shown above (Fig. 1D), this strain had an elevated $\beta$-galactosidase level due to the absence of intrinsic autoregulatory control. Cell cultures were grown up to $\mathrm{OD}_{600} \sim 0.35$, then half the culture was treated with TPEN to induce the YkgM synthesis (see Fig. 2B). In 2 h of cultivation, treated and untreated cells were harvested, and $\beta$-galactosidase activities were measured in clarified cell lysates. As shown on Figure 2C, the $\beta$-galactosidase activity in cells treated with TPEN is reduced as compared with the untreated samples to the level 

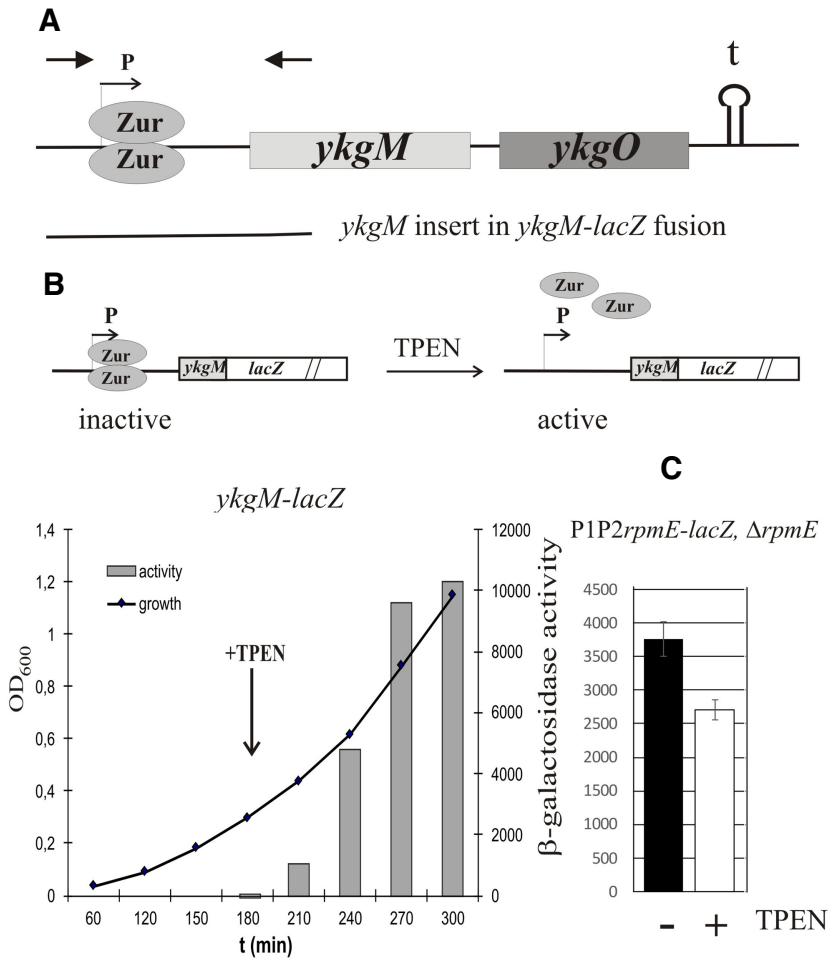

FIGURE 2. Expression of the $y \mathrm{kgM}$ gene upon induction of zinc starvation. (A) Structure of the $y k g M-y k g O$ operon. Region amplified to generate the $y \mathrm{kgM}$-lacZ fusion is designated by a line below the operon structure. $(\mathrm{P})$ - promoter, $(\mathrm{t})$ - terminator, primers used for PCR are indicated by arrows, Zur-transcriptional Zn-dependent repressor functioning as a dimer. (B) Induction of the $y \mathrm{kgM}$-lacZ expression by treating the growing culture with TPEN. (C) Effect of the ykgM induction by TPEN on the P1P2rpmE-lacZ expression in a bL31-deficient strain.

measured for the rpmE ${ }^{+}$-strain (see Fig. 1B, P1P2/ pACYC184). Although indirectly, this may suggest that YkgM is able not only to replace bL31 in ribosome structure (Lilleorg et al. 2019) but also to modulate the rpmE expression.

\section{Comparative structural analysis of the rpmE $5^{\prime}$-UTRs from $\gamma$-proteobacteria}

The pivotal role of the conserved secondary structures within $5^{\prime}$-UTRs of mRNAs in translation regulation of r-protein operons has been increasingly recognized (Allen et al. 1999, 2004; Boni et al. 2001; Tchufistova et al. 2003; Aseev et al. 2008, 2009; Fu et al. 2013, 2014; Meyer 2017, 2018; Mustoe et al. 2018). To compare secondary structures of the rpmE 5'UTRs from a representative set of $\gamma$-proteobacterial species (see Material and Methods), first we outlined presumable promoters corresponding to P2 of E. coli which is a typical $\sigma^{70}$ promoter (Schultzaberger et al. 2007). The sequences upstream of the rpmE coding frame were visually inspected for the presence of the -10 hex- amer, and the presumable promoter regions were analyzed by sequence logo analysis (Crooks et al. 2004). The found conservation of patterns for -10 and -35 promoter regions and an optimal spacing in between (Fig. 3A) allowed us to define the transcription start sites (assumed to be located 7 bp downstream from the -10 hexamer) and hence the length of 5'-UTRs with a high probability.

Computer modeling of putative secondary structures of the rpmE 5'-UTRs from different families of $\gamma$-proteobacteria (Zuker 2003; Bon et al. 2012) and analysis of their conservation revealed a highly conserved stem-loop structure preceding the rpmE ribosome binding site (Fig. 3B-E). In all species analyzed, this long irregular hairpin bears two nearly situated bulges, B1 and B2 (encircled on Fig. 3B, C). While the unpaired G-residues in B1 represent a universal feature, B2 varies depending on species analyzed, and based on its structure the conserved hairpins can be divided into two groups further referred to as E. coli-like (Fig. 3B,D) and Yersinia-like (Fig. 3C,E). E. coli-like B2 comprises a G-residue on one side and an unpaired AGA sequence (AUG in Providencia) on the other side, whereas all Yersinia-like stem-loops (Fig. 3C,E) bear only one unpaired G-residue in B2. Remarkably, in all species belonging to the family Enterobacteriaceae that embraces the closest relatives of E. coli, the B1/B2-containing stemloops are not only conserved, they are identical at the sequence level, which emphasizes their functional validity. At the same time, the family Morganellaceae comprises species belonging to both groups: In the genus Providencia, the rpmE stem-loop belongs to the E. coli-like type (Fig. 3D), while in other genera (Proteus, Photorhabdus, Morganella, Xenorhabdus) to the Yersinia-like group (Fig. $3 E)$. It should be noted that the Yersinia-like group includes the Pseudomonas species, which is a rare case among conserved regulatory structures controlling r-protein operons, viz. rpsB (Aseev et al. 2008, 2009) or rpsF (Fu et al. 2014; Babina et al. 2015), while most others demonstrate more narrow distribution (Allen et al. 1999, 2004; Tchufistova et al. 2003; Fu et al. 2013; Aseev et al. 2015; Meyer 2018). Importantly, the structure proposed here for the E. coli rpmE 5'UTR (Fig. 3B) exists in living E. coli cells during mid-log growth as has been shown by the SHAPE-MaP approach, a chemical probing strategy to study RNA structures across the E. coli transcriptome (Mustoe et al. 2018, Anthony Mustoe personal communication). Moreover, in growing cells, a small hairpin comprising the SD sequence (Fig. 3B) was not detected, and 5'-UTR downstream from the operator stem-loop was completely unstructured, consistent with the active translation initiation, while the SDcontaining hairpin was indeed formed when translation initiation was inhibited by kasugamycin treatment (Anthony Mustoe personal communication).

To test whether the difference in a structure of $B 2$ is essential for feedback regulation, we created a recombinant 
A

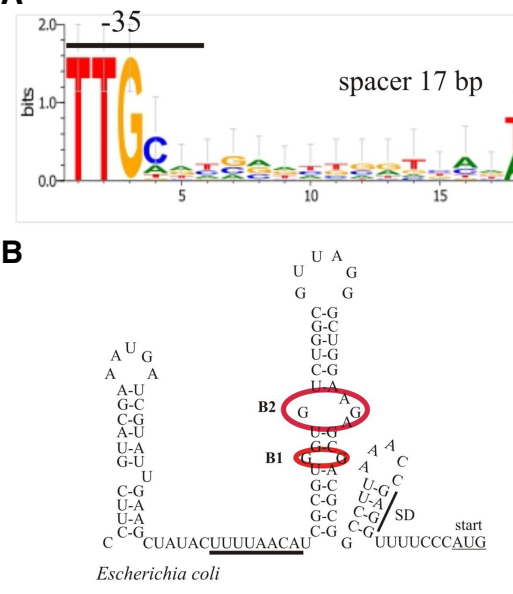

D
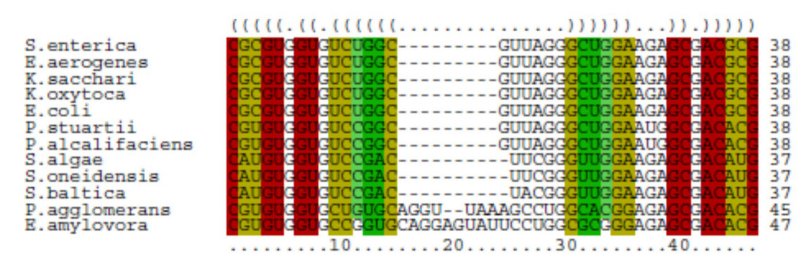

$\mathbf{E}$
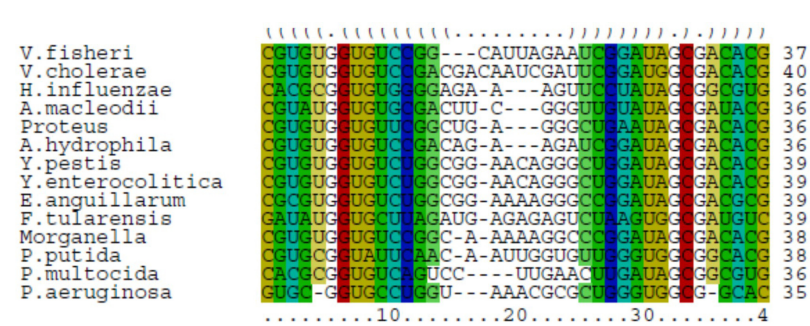

$\mathbf{F}$

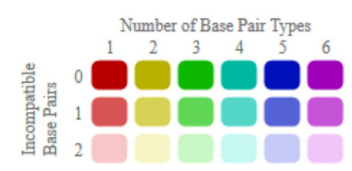

Compatible base pairs are colored, where the hue shows the number of different types C-G, G-C, A-U, U-A, G-U or U-G of compatible base pairs in the corresponding columns. In this way the hue shows sequence conservation of the base pair. The saturation decreases with the number of incompatible base pairs. Thus, it indicates the structural conservation of the base pair.

FIGURE 3. Conservation of the regulatory structure of the rpmE mRNA in $\gamma$-proteobacteria. (A) Conservation of the rpmE promoter patterns (corresponding to the rpmE promoter P2 of E. coli) in $\gamma$-proteobacteria, which allows defining TSSs and $5^{\prime}$-UTRs. Sequence logo analysis of the rpmE promoter regions from various species listed in Materials and Methods. (B) Putative secondary structure of the E. coli rpmE 5'-UTR in a transcript from the P2 promoter. Bulges B1 and B2 in a stem-loop preceding RBS are encircled, an AU-rich sequence in front of the conserved stem-loop is underlined. (C) Putative secondary structure of the $Y$. pestis rpmE $5^{\prime}$-UTR. B1 and B2 are encircled. $(D, E)$ Conservation of the presumable autogenous operator for E. coli-like $(D)$ and Yersinia-like $(E)$ structures as revealed by LocARNA algorithm (Smith et al. 2010). (F) Color legend for $D$ and $E$.

E. coli strain bearing the rpmE regulatory regions from $Y$. pestis fused in frame with the chromosomal lacZ gene. The strain was then transformed with pL31 and an empty vector as a control. While the $\beta$-galactosidase assays showed the bL31-mediated decrease in the expression of the Yersinia-derived reporter, the extent of repression was lower than in the case of the authentic regulatory regions from E. coli (Fig. 4A). This may indicate the possible contribution of the B2 structure to the autogenous control.

\section{Site-directed mutagenesis of a presumable bL31 operator site}

To examine the role of the bulges B1 and $\mathrm{B} 2$ in autogenous regulation of the rpmE expression in E. coli, we used site-directed mutagenesis. We first eliminated the universal bulge B1 (unpaired G-residues) shared by both E. coli-like and Yersinia-like stem-loops by changing one $\mathrm{G}$ for a C-residue in both rpmE-lacZ reporters (driven by P1P2-promoter regions and by $\mathrm{P} 2$ alone). The mutation was initially introduced in the corresponding plasmids (Table 1) and then transferred onto the chromosome by homologous recombination. The $\beta$ galactosidase assay revealed the significant alleviation of a negative impact of pL31 on the mutant (G-to-C) P1P2-reporter and its disappearance in the case of the mutant P2-construct (Fig. 4B), indicating that $\mathrm{B} 1$ is essential for the autogenous control.

The second mutation was aimed to remove B2 specific for Enterobacteriaceae. We substituted AGA for a C, thereby transforming the bulge B2 into a canonical $\mathrm{G}-\mathrm{C}$ base pair. The $\beta$-galactosidase assay showed an abolishment of the autogenous control, and in addition, a significant increase in the expression level of both P1P2- and P2rpmE-lacZ reporters (Fig. 4C). The fact that both reporters show parallel changes in activities caused by the $\Delta \mathrm{B} 2$ mutation suggests that the remote upstream region (the beginning of the $\mathrm{P} 1$ transcript) plays a minimal role in autogenous regulation of the rpmE expression. Thus, phylogenetic analysis of the secondary structure conservation (Fig. 3D,E) combined with site-directed mutagenesis allowed us to define 
the conserved bulges B1 and B2 as key structural elements ensuring the autogenous control of the rpmE expression.

\section{Translation initiation region of the rpmE mRNA is noncontiguous}

A significant increase in translation activities of both reporters caused by the $\Delta \mathrm{B} 2$ mutation seems, at first glance, surprising, because this mutation leads to a considerable increase in the hairpin structure stability, with $\Delta \mathrm{G}$ changing from $-16.0 \mathrm{kcal} / \mathrm{mol}$ to $-23.7 \mathrm{kcal} / \mathrm{mol}$. The presence of stable secondary structures in the vicinity of the ribosome binding site (RBS) is generally considered as a negative factor affecting translation efficiency (De Smit and Van Duin 1994). However, the existence of noninhibitory or even positive structural elements in translation initiation regions (TIRs) of prokaryotic mRNAs has been repeatedly reported (Gold 1988; Sacerdot et al. 1998; Nivinskas et al. 1999; Boni et al. 2001; Nafissi et al. 2012). According to these data, the ribosomal mRNA binding track can tolerate the presence of long stable stem-loop structures that may optimally arrange crucial mRNA elements involved in initiation complex formation. Thus, the RBSs of the T4 genes 38 (Gold 1988) and 25 (Nivinskas et al. 1999) comprise stable hairpins separating the SD sequence from the start codon. It was suggested that they positively affect translation initiation efficiency by bringing these two essential elements into necessary proximity, without interfering with joining of the $50 \mathrm{~S}$ subunit. RBSs or TIRs of this type may be called noncontiguous or split.

Another case, which bears more close resemblance to the rpmE TIR, was described for the threonyl-synthetase mRNA. The translation efficiency of the thrS mRNA is provided not only by an SD-sequence properly distanced from the start codon, but also by a single-stranded upstream region separated from the SD-element by a strong stem-loop structure which serves as an autogenous operator rec- ognized by ThrS (Sacerdot et al. 1998). The rpmE 5'-UTR structure also comprises a rather long, AU-rich singlestranded (ss) region in front of the conserved operator stem-loop (Fig. 3B). We believe that this ss-region may act as a "standby" site (Sterk et al. 2018) or as a translational enhancer responsible for the increase in the rpmE TIR
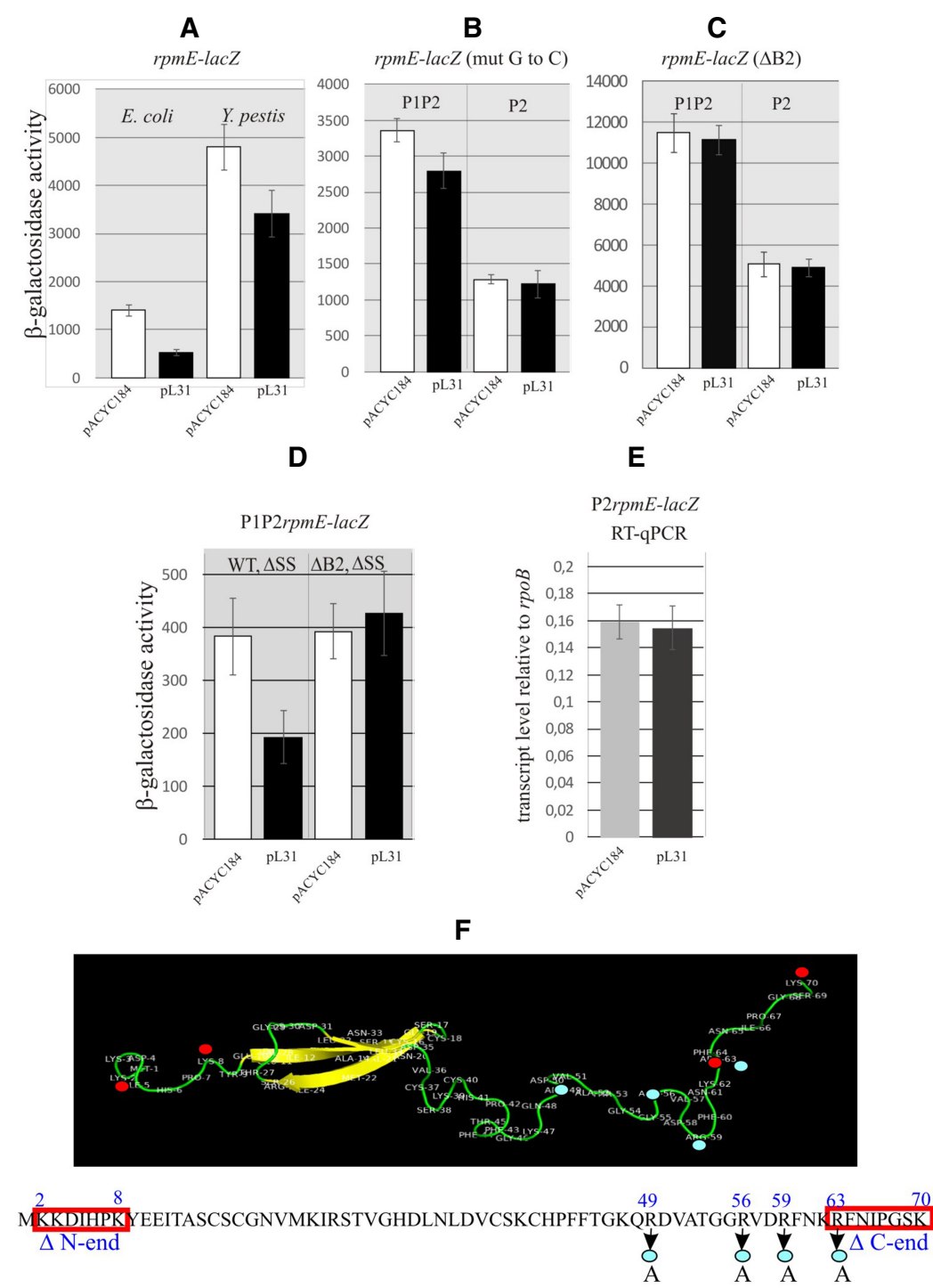

\begin{tabular}{|lcccccc|}
\hline $\begin{array}{l}\text { mutation } \\
\text { repression }\end{array}$ & $\begin{array}{c}\Delta \text { C-end } \\
\text { yes }\end{array}$ & $\begin{array}{c}\text { R63A } \\
\text { yes }\end{array}$ & $\begin{array}{c}\text { R59A } \\
\text { yes }\end{array}$ & $\begin{array}{c}\text { R56A } \\
\text { yes }\end{array}$ & $\begin{array}{c}\text { R49A } \\
\text { yes }\end{array}$ & $\begin{array}{c}\Delta \text { N-end } \\
\text { no }\end{array}$ \\
\hline
\end{tabular}

G

$\beta$-galactosidase activity dot-blot assay
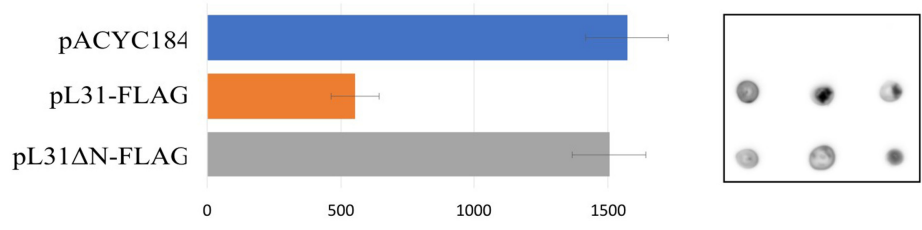

FIGURE 4. (Legend on next page) 
activity in the $\triangle \mathrm{B} 2$ mutant variant. In the wild-type rpmE $T I R$, the conserved stem-loop is less rigid, and the bulges B2/B1 may be spatially arranged in a manner allowing their direct contacts with the initiating ribosome.

To validate our assumption that the upstream ss-region may act as a translational enhancer, we deleted the UUUUAACAU-sequence (underlined on Fig. 3B) in front of the conserved stem-loop both from the wild-type P1P2rpmE-lacZ reporter and from its $\Delta \mathrm{B} 2$ mutant (Materials and Methods). In both cases, we observed a great decrease in the $\beta$-galactosidase activities (Fig. 4D), which indicates that this ss-region does enhance translation efficiency, presumably via its interaction with the rprotein S1 upon formation of the 30S initiation complex (see Komarova et al. 2002, 2005; Romilly et al. 2019). This is in line with our idea that the rpmE TIR represents a case of a noncontiguous TIR. It is noteworthy, while the wild-type P1P2rpmE-lacZ construct bearing a 9-bp deletion retained its susceptibility to the autogenous control, the double mutant was not sensitive to the presence of bL31 in trans just as its $\Delta B 2$ parent. This gives additional arguments in favor of an essential role of B2 in autogenous control of the rpmE expression.

\section{The rpmE feedback regulation occurs at the translation level}

To verify that bL31 regulates its own expression at the translation level, we compared the transcript yields for the P2rpmE-lacZ reporter in cells bearing PL31 and an empty vector by using RT-qPCR with an internal standard (Materials and Methods). We did not find any significant difference in transcript amounts which could denote other possible control mechanisms like preliminary transcription termination or a decrease in transcript stability (Fig. 4E). This led us to conclude that the rpmE expression is regulated at the level of translation.

\section{Possible mechanisms for translational autogenous regulation of the rpmE gene}

bL31 represents an unusual translational repressor, because in ribosomes, it is involved in formation of the protein-protein intersubunit bridge B1b through interactions with L5 in a central protuberance of the $50 \mathrm{~S}$ subunit and with $\mathrm{S} 13$ in a head of 30S (Fischer et al. 2015; Liu and Fredrick 2016), whereas its site-specific interactions with rRNA have not been defined. At the same time, the recently proposed TriPepSVM algorithm able to predict RNA-binding features rely- ing on the primary structure only catalogued bL31 as an RNA-binding protein (RBP) in E. coli and Salmonella (Bressin et al. 2019), which is in line with its repressor activity discovered by us. Next, since the spatial structure of a free bL31 has remained undetermined, we used an advanced web server IntFOLD for high performance 3D structure prediction (McGuffin et al. 2019). According to the model obtained (Fig. 4F), bL31 in a free state is largely disordered and does not contain any classical RNA-binding fold. Importantly, many of the recently identified RBPs do not harbor canonical RNA-binding domains, but may achieve RNA binding through sequences that are intrinsically disordered and often of low amino acid complexity (Calabretta and Richard 2015; Järvelin et al. 2016; Ntountoumi et al. 2019). Thus, we considered the possibility that the role of bL31 as an autogenous repressor may rely on its ability to directly interact with the operator stem-loop through its intrinsically disordered regions.

To find out which part of a highly disordered bL31 (Fig. 4F) participates in autogenous regulation, we mutated the rpmE coding region within the plasmid pL31. All the mutated variants obtained were sequenced and then analyzed for their ability to inhibit expression of the P2rpmE-lacZ reporter. First, we deleted the carboxy-terminal part of the protein (amino acid residues 63 to 70 ) essential for the interaction of bL31 with the $30 S$ subunit (Ueta et al. 2017). In particular, Arg63 was shown to interact with the phosphate backbone of the 16S rRNA helix h42 (A1311 and G1312) (Fischer et al. 2015; Liu and Fredrick 2016). The resulting plasmid $\mathrm{pL} 31 \Delta \mathrm{C}$ was still able to inhibit the rpmE-lacZ expression to the same extent as the initial pL31 expressing wild-type bL31. Then, we mutated several arginines in a region (residues 41 to 59) which was predicted to possess an RNA-binding activity by the TriPepSVM algorithm (Bressin et al. 2019). We substituted R49, R56, and R59 + R63 for alanines, but none of these changes impaired the repressor activity of
FIGURE 4. Distinctive features of the rpmE expression regulation in vivo. (A) Comparison of the bL31-mediated repression levels for $E$. coli- and Y. pestis-derived rpmE-lacZ reporters. $(B, C)$ Site-directed mutations of $B 1(B)$ and $B 2(C)$ in $E$. coli rpmE-lacZ reporters under the control of both P1P2 and P2-only promoters eliminate autogenous repression by bL31 in trans. (D) Deletion of a single-stranded region ( $\triangle \mathrm{SS}$ ) upstream of the operator stem-loop greatly reduces translation efficiency of the P1P2rpmE-lacZ reporter but does not affect autogenous control. (E) Comparison of P2rpmE-lacZ transcript amounts in cells bearing an empty vector or its derivative PL31 suggests that autogenous repression occurs at the translation level. The results of RT-qPCR analysis with the internal control (rpoB). ( F) Mutational analysis of bL31 produced in trans reveals the necessity of the unstructured amino-terminal region (but not the carboxy-terminal part) for its repressor activity. Primary and presumable 3D structures predicted by IntFOLD (McGuffin et al. 2019) are shown. Deletions are boxed in the bL31 sequence, and the first and the last deleted residues are marked with red circles on the 3D structure. Positions of point mutations ( $R$ to $A$ ) are marked with blue circles. (G) FLAG-tagging of bL31 and bL31 $\Delta \mathrm{N}$ does not change their ability (bL31-FLAG) or inability (bL31 $\Delta \mathrm{N}-\mathrm{FLAG}$ ) to act as an autogenous repressor. The results of the $\beta$-galactosidase assay are shown. The dot-blot immunoassay with the anti-FLAG antibody M2 revealed that the FLAG-tagged proteins were synthesized in comparable amounts. In both assays, three independent biological replicates were tested. 
bL31 (Fig. 4F). These results suggest that the part of bL31 involved in interactions with the $30 \mathrm{~S}$ subunit is not responsible for the autoregulatory function. Finally, we deleted the amino-terminal unstructured region (residues 2 to 8). The corresponding plasmid $\mathrm{pL} 31 \Delta \mathrm{N}$ completely lost an ability to inhibit the rpmE-lacZ expression, indicating that its product, L31 lacking the lysine-rich disordered amino terminus, was unable to serve as an autogenous repressor, presumably due to a loss of RNA-binding specificity. We should note that $\mathrm{pL} 31 \Delta \mathrm{N}$ has also revealed a partially decreased ability to suppress the slow-growth phenotype of the $\Delta$ rpmE mutant (not shown), most likely due to a decreased affinity to the $50 \mathrm{~S}$ subunit during ribosome assembly.

Since the carboxyl terminus is not essential for the regulatory activity of bL31, we modified the plasmids pL31 and pL31 $\Delta \mathrm{N}$ to provide the carboxy-terminal FLAG-tagging of the expressed proteins. The presence of the FLAG epitope did not change an ability of bL31 to inhibit the rpmE-lacZ expression, with the $L 31 \Delta \mathrm{N}-\mathrm{FLAG}$ remaining unable to act as a repressor (Fig. 4G). To compare the amounts of proteins expressed from pL31wt and $\mathrm{pL} 31 \Delta \mathrm{N}$ in a cell, we used the dot-blot immunoassay with the anti-FLAG M2 monoclonal antibody. The results showed that L31wtFLAG and L31 $1 \mathrm{~N}-\mathrm{FLAG}$ were synthetized in comparable amounts (though the amount of $L 31 \Delta N-F L A G$ was somewhat lower), suggesting that the absence of the $L 31 \Delta \mathrm{N}$ mediated repression is not due to its highly diminished synthesis in a cell (Fig. 4G).

It remains still unresolved how RNA-binding specificity of RBPs can be attained through intrinsically disordered regions (see Järvelin et al. 2016). In the case of bL31, we cannot exclude that its protein partners in the ribosome might help its repressor activity. First of all, it concerns $L 5$ that interacts with the amino-terminal domain of bL31, while the disordered amino-terminal residues may contact 5S rRNA (Fischer et al. 2015). So far RNA-binding mediated by intrinsically disordered regions has been described mainly for numerous eukaryotic proteins (Calabretta and Richard 2015; Järvelin et al. 2016). In this regard, bL31 represents an intriguing example of a prokaryotic, largely disordered protein demonstrating an RNA-binding specificity.

\section{Concluding remarks}

The important role of the autogenous control of r-protein synthesis in coordinated production of ribosomal components has been repeatedly underlined. Moreover, recent data indicate this mechanism is necessary for overall organism viability (Babina et al. 2018). As mentioned above, the most part of $E$. coli r-protein operons have been already examined (16 out of 21), and only two of them, rp/U-rpmA and rpmB-rpmG, did not reveal any impact of the excess r-protein production on the expression level of the operon-specific reporters (Aseev et al. 2016). In this study, we identified one more regulatory loop, viz. the modulation of the rpmE gene expression by its product, bL31, an essential component of the intersubunit bridges $\mathrm{B} 1 \mathrm{~b} / \mathrm{c}$. The rpmE translation regulation shows both typical and peculiar features. The feature typical of the most part of described cases is the presence of a highly conserved cis-regulatory RNA structure in a $5^{\prime}$ UTR, which serves as a translational operator, an indispensable attribute of the autogenous control mediated by r-proteins (for reviews, see Fu et al. 2013; Meyer 2017, 2018). The distinctive feature is that the translational initiation region (TIR) of the rpmE $m$ RNA represents a rare case of a noncontiguous $T I R$, where a stable operator stem-loop structure separates an AU-rich translational enhancer from a Shine-Dalgarno element; to our knowledge, this type of a noncontiguous TIR has been previously described only for the thrS mRNA (Sacerdot et al. 1998). Another peculiar feature of the rpmE regulation is the nature of the trans-acting r-protein-repressor, bL31, whose function on a ribosome is to take part in the formation of the protein-protein bridge B1b, while its site-specific interactions with rRNA have not been defined. Nevertheless, recent computational tools catalog bL31 as an RNA-binding protein (Bressin et al. 2019; Ntountoumi et al. 2019), which is consistent with its repressor activity found here. The mutational analysis revealed that an intrinsically disordered amino-terminal region of bL31 likely contributes to its repressor function. We believe the mechanistic details of the bL31-mediated autogenous control represent an intriguing task for future studies.

\section{MATERIALS AND METHODS}

\section{Bacterial strains and plasmids}

Strains and plasmids used in this work are listed in Table 1. The plasmid pL31 was created by cloning the PCR fragment corresponding to the entire rpmE gene flanked with its own promoters and terminator into BamHI/HindIII sites of pACYC184. The restriction sites were included in the structure of the forward and reverse primers (marked on Fig. 2A). The structure of pL31 was checked by sequencing, and expression of the wild-type bL31 from the plasmid was verified by its ability to suppress the slow-growth phenotype of the rpmE::kan strain JW3907 (Baba et al. 2006).

The rpmE-lacZ chromosomal fusions were generated as previously described (Aseev et al. 2008, 2009, 2015, 2016). DNA fragments corresponding to the rpmE region including promoters, $5^{\prime}$-UTR and the beginning of the coding sequence were amplified from the genomic DNAs of E. coli or $Y$. pestis by using primers containing BamHI and HindIII sites for cloning into pEMBL $\Delta 46$ (Dreyfus 1988) in-phase with the lacZ sequence. The $y \mathrm{kgM}-\mathrm{lacZ}$ fusion was constructed in the same way. The plasmids were first used to transform $\mathrm{DH} 5 \alpha$ cells, and proper clones were selected by $\alpha$-complementation. Plasmids were isolated from blue colonies, sequenced and used for transformation of the $\mathrm{Lac}^{-}$strain ENSO (Dreyfus 1988, former name HfrG6 $\Delta 12$ ) and subsequently transferring the reporter fusions onto the chromosome by homologous recombination, selecting for $\mathrm{Lac}^{+}$phenotype on 
McConkey agar. The rpmE::kan allele from JW3907 and zur::kan from JW5714 (Baba et al. 2006) were transferred into strains bearing the rpmE-lacZ and $y \mathrm{kgM}$-lacZ reporters, respectively, by P1 transduction.

\section{Growth of cells and $\beta$-galactosidase assays}

Strains were grown at $37^{\circ} \mathrm{C}$ in Luria-Bertani (LB) medium supplemented with antibiotics if necessary: kanamycin (kan) $30 \mu \mathrm{g} / \mathrm{mL}$, chloramphenicol $(\mathrm{Cm}) 34 \mu \mathrm{g} / \mathrm{mL}$, harvested in exponential phase at an optical density at $600 \mathrm{~nm}\left(\mathrm{OD}_{600}\right)$ about $0.4-0.5$ and used for preparing clarified cell lysates. The cells were broken by a repeated thawing-freezing procedure, the lysates were treated with the RNase-free DNase and clarified by centrifugation as previously described (Tchufistova et al. 2003). Protein concentration in a fraction of soluble proteins was determined by Bradford assay (Bio-Rad). Specific $\beta$-galactosidase activities in the same fraction were measured according to Miller (1972) and expressed in nmol ONPG (o-nitrophenyl- $\beta$-D-galactopyranoside) hydrolyzed per minute per milligram of total soluble cell proteins.

\section{Zinc deprivation experiments}

The zinc chelator TPEN [N,N,N,N-tetrakis(2-pyridyl-methyl)] ethylenediamine (Sigma-Aldrich) was used to induce $\mathrm{Zn}^{2+}$-starvation in growing cultures (Sigdel et al. 2006; Hensley et al. 2012). Cells harboring the $y \mathrm{kgM}-\mathrm{lacZ}$ chromosomal fusion were grown at $37^{\circ} \mathrm{C}$ to mid-log phase $\left(\mathrm{OD}_{600} \sim 0.4\right)$ in $\mathrm{M9}$ minimal medium supplemented with Casamino acids $(10 \mathrm{~g} / \mathrm{L}), 1 \mathrm{mM} \mathrm{MgSO} 47 \mathrm{H}_{2} \mathrm{O}$, $0.1 \mathrm{mM} \mathrm{CaCl} 2,0.2 \%$ glycerol, $1 \mu \mathrm{g} / \mathrm{mL}$ thiamin (Miller 1972) and then treated with $5 \mathrm{mM}$ TPEN solution in $50 \%$ ethanol (final concentration $10 \mu \mathrm{M}$ ). Aliquots of $3 \mathrm{~mL}$ were withdrawn just before and 30,60, 90, and 120 min after the addition of TPEN, placed on ice, and lysed as described above for preparing cell lysates for the $\beta$-galactosidase assay. To test the possibility of modulating the rpmE-lacZ expression by YkgM (as a paralog of bL31), we used the bL31-deficient strain bearing the P1P2rpmE-lacZ chromosomal reporter (Table 1). The cells were grown in M9 medium described above to $\mathrm{OD}_{600} \sim 0.35$, then cultures were divided in half, and one portion was treated by TPEN to induce the YkgM synthesis. Treated and nontreated cultures were cultivated for $2 \mathrm{~h}$, placed on ice, and cell lysates were prepared for the $\beta$-galactosidase assay.

\section{Site-directed mutagenesis}

For site-directed mutagenesis we used two pEMBL $\triangle 46$-derivatives bearing the $E$. coli rpmE-lacZ fusions driven by the entire rpmE promoter region (pEP1P2rpmE-lacZ) or by the promoter P2 alone (pEP2rpmE-lacZ). First, we mutated the universal B1 bulge (two unpaired $G$ residues) in the conserved stem-loop structure (located upstream of the rpmE RBS) by changing the $G$ residue on the left side of the hairpin for a $C$, thus transforming B1 into a canonical C-G pair. To this end, the two-step PCR technique was used. At the first step, two overlapping PCR fragments were obtained on each plasmid with two pairs of primers: rpmE_GtoC-for (5'-CATCGCGTCGTGTCTGGCGTTAG) together with DSlac, and rpmE_GtoC-rev (5'-CTAACGCCAGACACGAC
GCGATG) together with UPlac (mutated base is underlined). UPlac and DSlac were previously described (Komarova et al. 2002). At the second step, the two purified PCR fragments were mixed and amplified in the presence of UPlac and DSlac. Finally, the resulting PCR fragments were treated with BamHI and HindIII and cloned into pEMBL $\Delta 46 / \mathrm{BamHI}$, HindIII to create pEP1P2rpmE-lacZ(GtoC) and pEP2rpmE-lacZ(GtoC). The plasmids were sequenced and used to transfer a " $G$ to $C$ " mutation onto the chromosome by homologous recombination.

Another PCR approach was used to create a mutation transforming the bulge B2 ( $G$ on the left side and AGA on the right side of the conserved hairpin, Fig. 3B) into a canonical G-C pair by changing AGA for a $C$. The following primers were designed to delete AGA by amplifying the whole plasmid (pEP1P2rpmElacZ or pEP2rpmE-lacZ) with the use of Phusion HotStartll DNA polymerase (Thermo Scientific): rpmE_delAGA-for 5'-GCGACG CGGCCTTAAACCG and rpmE_delAGA-rev 5'-GTCCAGCCCTA ACGCCAGAC. The reverse primer had a $G$ at the $5^{\prime}$-end to provide formation of a G-C pair in the final product. The PCR products of proper lengths were purified, treated with T4-polynucleotide kinase in the presence of cold ATP, and after inactivating the kinase $\left(10 \mathrm{~min}\right.$ at $\left.70^{\circ} \mathrm{C}\right)$ the blunt ends were ligated. The ligated products were purified, treated with Dpnl, and used to transform $\mathrm{DH} 5 \alpha$. The resulting plasmids (pEP1P2rpmE-lacZ_ $\Delta \mathrm{B} 2$ and pEP2rpmE-lacZ_ $\Delta$ B2) were sequenced and then used to transfer the " $\Delta \mathrm{B} 2$ " mutation onto the chromosome of ENSO by homologous recombination. The deletion of a 9-bp fragment from the AT-rich region preceding the conserved stem-loop was done in a similar way with primers rpmE_delAT-for 5'-CGCGTGTGTC TGGCGTTAG and rpmE_delAT-rev 5'-GTATAGCTTCAATAC GATCATTTCGTAC. At the mRNA level, the deleted sequence is underlined in Figure $3 \mathrm{~B}$. The deletion was introduced both in pEP1P2rpmE-lacZ and in pEP1P2rpmE-lacZ_AB2 plasmids, and then transferred onto the chromosome, resulting in mutant strains LAB_P1P2rpmE::lacZ_Ass, and LAB_P1P2rpmE::

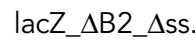

\section{RT-qPCR}

Total RNA was isolated from exponential cultures of LAB_P2rpmE::IacZ bearing pL31 or an empty vector using the RNeasy Mini Kit (Qiagen) according to recommendations of the manufacturer. RNase-free DNase (Qiagen) was added to the columns during RNA extraction for 15 min to ensure the absence of DNA contamination in RNA samples. The amount of total RNA in preparations was estimated by measuring the $\mathrm{OD}_{260}$. Reverse transcription (RT) was performed with AMV reverse transcriptase (Promega) in final volume $20 \mu \mathrm{L}$ for $1 \mathrm{~h}$ at $42^{\circ} \mathrm{C}$ on $1 \mu \mathrm{g}$ of total RNA in the presence of two reverse primers $(1 \mu \mathrm{L}$ of $5 \mu \mathrm{M}$ solution each) corresponding to lacZ (DSlac, see Komarova et al. 2002) and to the reference gene rpoB (rpoB-rev: 5'-CGGATTTGACATT CCTGGACGTC). Real-time PCR (qPCR) was run with the use of LightCycler 96 (Roche); each $25 \mu \mathrm{L}$ reaction contained $2 \mu \mathrm{L}$ RT mix, $5 \mu \mathrm{L} 5 \times$ qPCRmix HS SYBR (Evrogen), and the following primers: rpmE_P2-for 5'-CTTCGTACGAAATGATCGTATTGAAGC, DSlac, rpoB-for 5'-ACGTCCACAAGTTCTGGATGTACC and rpoB-rev ( $1 \mu \mathrm{L}$ of $5 \mu \mathrm{M}$ solution each). Two independently isolated preparations of total RNA were used for RT, and three technical replicates for each $\mathrm{QPCR}$ reaction were run simultaneously. 
Control qPCR reactions without RT were done to exclude DNA contaminations in RNA preparations. LinRegPCR software was used to quantify transcript amounts relative to the reference transcript rpoB.

\section{Mutational analysis of bL31}

To search for the bL31 region required for its role as an autogenous repressor, we mutated the rpmE coding part within the plasmid pL31. First, we deleted the carboxy-terminal tail, which contributes to ribosome formation and translational activity (Ueta et al. 2017). To this end, we used PCR with primers flanking the sequence to be deleted to amplify the whole pL31 except for the region encoding the $\mathrm{C}$-tail (residues 63 to 70). The same approach was used for deleting the amino-terminal part of bL31 (residues 2 to 8 ). In both reactions, Phusion HotStartll DNA polymerase (Thermo Scientific) was used, and protocols were essentially the same as described above for the $\Delta B 2$ deletion. In addition, we introduced point mutations by changing several arginines (encoded by CGT triplets) for alanines (GCT). To this end, the two-step PCR technique was used (see above). At first step, two PCR fragments were obtained with "internal" overlapping primers comprising mutations and "external" primers bearing BamHI (forward) and HindIII (reverse) sites which were used for cloning the rpmE gene in PACYC184 (Fig. 1A). At the second step, the two PCR products were mixed and amplified in the presence of "external" primers; next, the products obtained were treated with BamHI and HindIII and ligated into PACYC184/ BamHI, HindIII. The resulting plasmids were sequenced to confirm the presence of the desired mutations in the rpmE gene.

\section{FLAG-tagging and dot-blot analysis of bL31wt and bL31 $\Delta \mathrm{N}$ expressed from $\mathrm{pL} 31 \mathrm{wt}$ and $\mathrm{pL} 31 \Delta \mathrm{N}$}

To provide the carboxy-terminal FLAG-tagging of the encoded proteins, plasmids $\mathrm{pL} 31 \mathrm{wt}$ and $\mathrm{pL} 31 \Delta \mathrm{N}$ were modified by inserting the FLAG-encoding sequence; the modification was done by Evrogen. The strain LAB_P2rpmE::lacZ was transformed with pL31wt-FLAG and pL31 $\triangle N-F L A G$, cells were grown to the midlog phase, and cell lysates were prepared as described above. The dot-blot immunoassay was performed as previously described (Hemm et al. 2010) with minor modifications. Aliquots of each sample (corresponding to the equal amount of $2 \mu \mathrm{g}$ of total soluble proteins) were spotted on a nitrocellulose membrane; the membrane was then dried at room temperature, blocked by incubating in $5 \%$ milk solution in phosphate-buffered saline with $0.4 \%$ Tween 20 (PBS-Tween) for $1 \mathrm{~h}$ and then probed with antiFLAG M2 monoclonal antibodies produced in mouse (SigmaAldrich), washed in PBS-Tween, and finally incubated with antimouse lgG HRP-linked secondary antibodies (Cell Signaling Technology). The results were visualized with Clarity Western ECL Substrate (Bio-Rad) and Bio-Rad VersaDoc MP4000 image station.

\section{Bioinformatic tools}

Nucleotide sequences of the rpmE gene and its flanking regions in various bacterial genomes were extracted from the NCBI Gene database. A graphical representation of the patterns within rpmE promoter sequences from a subset of $\gamma$-proteobacterial species belonging to different families was generated by using WebLogo (Crooks et al. 2004). The genomes analyzed were: E. coli, Salmonella enterica, Enterobacter aerogenes, Pantoea agglomerans, Providencia stuartii, Providencia alcalifaciens, Erwinia amylovora, Klebsiella oxytoca, Xenorhabdus bovienii, Photorhabdus temperate, Shewanella oneidensis, Shewanella algae, Proteus mirabilis, Morganella morganii, Dickeya dadantii, Aeromonas hydrophila, Alteromonas nucleodii, Pasteurella multocida, Haemophilus influenzae, Yersinia pestis, Yersinia enterocolitica, Vibrio fisheri, Vibrio cholerae, Francisella tularensis, Serratia fonticola, Pseudomonas syringae, $P$. aeruginosa, $P$. putida. Secondary structures of the rpmE $5^{\prime}$-UTRs were predicted by using algorithms Mfold (Zuker 2003) and McGenus (Bon et al. 2012). To demonstrate the conservation of the stem-loop structure in the rpmE 5'-UTR, the LocARNA algorithm (Smith et al. 2010) was used. The predicted 3D structure of a free bL31 was built with the use of IntFOLD web server (McGuffin et al. 2019). RNA-binding properties of bL31 were predicted by TriPepSVM algorithm (Bressin et al. 2019).

\section{ACKNOWLEDGMENTS}

We thank Petr Sergiev for sharing strains and Eliane Hajnsdorf for providing unpublished data on dRNA-seq for the rpmE gene. We are indebted to Anthony Mustoe for his interest in our results and for providing us with his unpublished data on the "in cell" secondary structure of the rpmE 5'UTR obtained by high-resolution SHAPE probing. We also express our gratitude to Artem Grigorov for his help with TriPepSVM algorithm. This work was supported by the RFBR grant 18-04-00743 and by the Russian Academy of Sciences.

Received December 5, 2019; accepted March 22, 2020.

\section{REFERENCES}

Akanuma G, Nanamiya H, Natori Y, Nomura N, Kawamura F. 2006. Liberation of zinc-containing L31 (RpmE) from ribosomes by its paralogous gene product, YtiA, in Bacillus subtilis. J Bacteriol 188: 2715-2720. doi:10.1128/JB.188.7.2715-2720.2006

Allen T, Shen P, Samsel L, Liu R, Lindahl L, Zengel JM. 1999. Phylogenetic analysis of L4-mediated autogenous control of the S10 ribosomal protein operon. J Bacteriol 181: 6124-6132. doi:10.1128/JB.181.19.6124-6132.1999

Allen TD, Watkins T, Lindahl L, Zengel JM. 2004. Regulation of ribosomal protein synthesis in Vibrio cholerae. J Bacteriol 186: 5933-5937. doi:10.1128/JB.186.17.5933-5937.2004

Aseev LV, Levandovskaya AA, Tchufistova LS, Skaptsova NV, Boni IV. 2008. A new regulatory circuit in ribosomal protein operons: S2mediated control of the rpsB-tsf expression in vivo. RNA 14: 1882-1894. doi:10.1261/rna.1099108

Aseev LV, Levandovskaya AA, Skaptsova NV, Boni IV. 2009. Conservation of regulatory elements controlling the expression of the rpsB-tsf operon in $\gamma$-proteobacteria. Mol Biol 43: 101107. doi:10.1134/S0026893309010142

Aseev LV, Bylinkina NS, Boni IV. 2015. Regulation of the rp/Y gene encoding 5 S rRNA binding protein L25 in Escherichia coli and related bacteria. RNA 21: 851-861. doi:10.1261/rna.047381.114 
Aseev LV, Koledinskaya LS, Boni IV. 2016. Regulation of ribosomal protein operons rp/M-rpsl, rpmB-rpmG, and rplU-rpmA at the transcriptional and translational levels. J Bacteriol 198: 2494-2502. doi:10.1128/JB.00187-16

Baba T, Ara T, Hasegawa M, Takai Y, Okumura Y, Baba M, Datsenko KA, Tomita M, Wanner BL, Mori H. 2006. Construction of Escherichia coli K-12 in-frame, single-gene knock-out mutant: the Keio collection. Mol Syst Biol 2: 2006.0008. doi:10.1038/ $\mathrm{msb} 4100050$

Babina AM, Soo MW, Fu Y, Meyer MM. 2015. An S6:S18 complex inhibits translation of E. coli rpsF. RNA 21: 2039-2046. doi:10.1261/ rna.049544.115

Babina AM, Parker DJ, Li GW, Meyer MM. 2018. Fitness advantages conferred by the L20-interacting RNA cis-regulator of ribosomal protein synthesis in Bacillus subtilis. RNA 24: 1133-1143. doi:10 $.1261 /$ rna.065011.117

Ban N, Beckmann R, Cate JH, Dinman JD, Dragon F, Ellis SR, Lafontaine DL, Lindahl L, Liljas A, Lipton JM, et al. 2014. A new system for naming ribosomal proteins. Curr Opin Struct Biol 24: 165169. doi:10.1016/j.sbi.2014.01.002

Bon M, Micheletti C, Orland H. 2012. McGenus: a Monte Carlo algorithm to predict RNA secondary structures with pseudoknots. Nucleic Acids Res 41: 1895-1900. doi:10.1093/nar/gks1204

Boni IV, Artamonova VS, Dreyfus M. 2000. The last RNA-binding repeat of the Escherichia coli ribosomal protein S1 is specifically involved in autogenous control. J Bacteriol 182: 5872-5879. doi:10 .1128/JB.182.20.5872-5879.2000

Boni IV, Artamonova VS, Tzareva NV, Dreyfus M. 2001. Non-canonical mechanism for translational control in bacteria: synthesis of ribosomal protein S1. EMBO J 20: 4222-4232. doi:10.1093/emboj/ 20.15.4222

Bressin A, Schulte-Sasse R, Figini D, Urdaneta EC, Beckmann BM M, Marsico A. 2019. TriPepSVM: de novo prediction of RNA-binding proteins based on short amino acid motifs. Nucleic Acids Res 47: 4406-4417. doi:10.1093/nar/gkz203

Calabretta S, Richard S. 2015. Emerging roles of disordered sequences in RNA-binding proteins. Trends Biochem Sci 40: 662-672. doi:10.1016/j.tibs.2015.08.012

Chadani Y, Niwa T, Izumi T, Sugata N, Nagao A, Suzuki T, Chiba S, Ito K, Taguchi H. 2017. Intrinsic ribosome destabilization underlies translation and provides an organism with a strategy of environmental sensing. Mol Cell 68: 528-539. doi:10.1016/j.molcel .2017.10.020

Crooks GE, Hon G, Chandonia J-M, Brenner SE. 2004. WebLogo: a sequence logo generator. Genome Res 14: 1188-1190. doi:10 $.1101 /$ gr. 849004

De Smit MH, Van Duin J. 1994. Control of translation by mRNA secondary structure in Escherichia coli. A quantitative analysis of literature data. J Mol Biol 244: 144-150. doi:10.1006/jmbi.1994 .1714

Dreyfus M. 1988. What constitutes the signal for the initiation of protein synthesis on Escherichia coli mRNAs? J Mol Biol 204: 79-94. doi:10.1016/0022-2836(88)90601-8

Eistetter AJ, Butler PD, Traut RR, Fanning TG. 1999. Characterization of Escherichia coli 50 S ribosomal protein L31. FEMS Microbiol Lett 180: 345-349. doi:10.1111/j.1574-6968.1999.tb08816.x

Fischer N, Neumann P, Konevega AL, Bock LV, Ficner R, Rodnina MV, Stark H. 2015. Structure of the E. coli ribosome-EF-Tu complex at $<3 \AA$ resolution by $\mathrm{C}_{\mathrm{s}}$-corrected cryo-EM. Nature 520: 567-570. doi:10.1038/nature14275

Fu Y, Deiorio-Haggar K, Anthony J, Meyer MM. 2013. Most RNAs regulating ribosomal protein biosynthesis in Escherichia coli are narrowly distributed to Gammaproteobacteria. Nucleic Acids Res 41: 3491-3503. doi:10.1093/nar/gkt055
Fu Y, Deiorio-Haggar K, Soo MW, Meyer MM. 2014. Bacterial RNA motif in the $5^{\prime}$ UTR of rpsF interacts with an S6:S18 complex. RNA 20: 168-176. doi:10.1261/rna.041285.113

Gilston BA, Wang S, Marcus MD, Canalizo-Hernández MA, Swindell EP, Xue Y, Mondragón A, O'Halloran TV. 2014. Structural and mechanistic basis of zinc regulation across the $E$. coli Zur regulon. PLoS Biol 12: e1001987. doi:10.1371/journal .pbio. 1001987

Gold L. 1988. Post-transcriptional regulatory mechanisms in Escherichia coli. Annu Rev Biochem 57: 199-233. doi:10.1146/ annurev.bi.57.070188.001215

Hemm MR, Paul BJ, Miranda-Ríos J, Zhang A, Soltanzad N, Storz G. 2010. Small stress response proteins in Escherichia coli: proteins missed by classical proteomic studies. J Bacteriol 192: 46-58. doi:10.1128/JB.00872-09

Hensley MP, Gunasekera TS, Easton JA, Sigdel TK, Sugarbaker SA, Klingbeil L, Breece RM, Tierney DL, Crowder MW. 2012. Characterization of $\mathrm{Zn}(\mathrm{II})$-responsive ribosomal proteins $\mathrm{YkgM}$ and L31 in E. coli. J Inorg Biochem 111: 164-172. doi:10.1016/j .jinorgbio.2011.11.022

Järvelin Al, Noerenberg M, Davis I, Castello A. 2016. The new (dis)order in RNA regulation. Cell Commun Signal 14: 9. doi:10.1186/ s12964-016-0132-3.

Komarova AV, Tchufistova LS, Supina EV, Boni IV. 2002. Protein S1 counteracts the inhibitory effect of the extended Shine-Dalgarno sequence on translation. RNA 8: 1137-1147. doi:10.1017/ S1355838202029990

Komarova AV, Tchufistova LS, Dreyfus M, Boni IV. 2005. AU-rich sequences within $5^{\prime}$-untranslated leaders enhance translation and stabilize mRNAs in Escherichia coli. J Bacteriol 187: 1344-1349. doi:10.1128/JB.187.4.1344-1349.2005

Le Derout J, Boni IV, Régnier P, Hajnsdorf E. 2010. Hfq affects mRNA levels independently of degradation. BMC Mol Biol 11: 17. doi:10 .1186/1471-2199-11-17

Lilleorg S, Reier K, Remme J, Liiv A. 2017. The intersubunit bridge B1b of the bacterial ribosome facilitates initiation of protein synthesis and maintenance of translational fidelity. J Mol Biol 429: 10671080. doi:10.1016/j.jmb.2017.02.015

Lilleorg S, Reier K, Pulk A, Liiv A, Tammsalu T, Peil L, Cate JD, Remme J. 2019. Bacterial ribosome heterogeneity: changes in ribosomal protein composition during transition into stationary growth phase. Biochimie 156: 169-180. doi:10.1016/j.biochi .2018.10.013

Liu Q, Fredrick K. 2016. Intersubunit bridges of the bacterial ribosome. J Mol Biol 428: 2146-2164. doi:10.1016/j.jmb.2016.02.009

Makarova KS, Ponomarev VA, Koonin EV. 2001. Two C or not two C: recurrent disruption of $\mathrm{Zn}$-ribbons, gene duplication, lineage-specific gene loss, and horizontal gene transfer in evolution of bacterial ribosomal proteins. Genome Biol 2: RESEARCH-0033. doi:10 .1186/gb-2001-2-10-reports0033

Mathy N, Pellegrini O, Serganov A, Patel DJ, Ehresmann C, Portier C. 2004. Specific recognition of rpsO mRNA and $16 \mathrm{~S}$ rRNA by Escherichia coli ribosomal protein S15 relies on both mimicry and site differentiation. Mol Microbiol 52: 661-675. doi:10 .1111/j.1365-2958.2004.04005.x

McGuffin LJ, Adiyaman R, Maghrabi AHA, Shuid AN, Brackenridge DA, Nealon JO, Philomina LS. 2019. IntFOLD: an integrated web resource for high performance protein structure and function prediction. Nucleic Acids Res 47: W408-W413. doi:10 .1093/nar/gkz322

Meyer MM. 2017. The role of mRNA structure in bacterial translational regulation. Wiley Interdiscip Rev RNA 8. doi:10.1002/wrna.1370.

Meyer MM. 2018. rRNA mimicry in RNA regulation of gene expression. Microbiol Spectr 6: RWR-0006-2017. doi:10.1128/ microbiolspecRWR-0006-2017 
Miller JH. 1972. Experiments in molecular genetics. Cold Spring Harbor Laboratory, Cold Spring Harbor, NY.

Mustoe AM, Busan S, Rice GM, Hajdin CE, Peterson BK, Ruda VM, Kubica N, Nutiu R, Baryza JL, Weeks KM. 2018. Pervasive regulatory functions of mRNA structure revealed by high-resolution SHAPE probing. Cell 173: 181-195. doi:10.1016/j.cell.2018.02 .034

Nafissi M, Chau J, Xu J, Johnson RC. 2012. Robust translation of the nucleoid protein Fis requires a remote upstream $A U$ element and is enhanced by RNA secondary structure. J Bacteriol 194: 2458-2469. doi:10.1128/JB.00053-12

Nivinskas R, Malys N, Klausa V, Vaiškunaite R, Gineikiene E. 1999. Post-transcriptional control of bacteriophage T4 gene 25 expression: mRNA secondary structure that enhances translational initiation. J Mol Biol 288: 291-304. doi:10.1006/jmbi.1999.2695

Nomura M, Gourse R, Baughman G. 1984. Regulation of the synthesis of ribosomes and ribosomal components. Ann Rev Biochem 53: 75-117. doi:10.1146/annurev.bi.53.070184.000451

Ntountoumi C, Vlastaridis P, Mossialos D, Stathopoulos C, lliopoulos I, Promponas V, Oliver SG, Amoutzias GD. 2019. Low complexity regions in the proteins of prokaryotes perform important functional roles and are highly conserved. Nucleic Acids Res 47: 999810009. doi:10.1093/nar/gkz730

Panina EM, Mironov AA, Gelfand MS. 2003. Comparative genomics of bacterial zinc regulons: enhanced ion transport, pathogenesis, and rearrangement of ribosomal proteins. Proc Natl Acad Sci 100: 9912-9917. doi:10.1073/pnas.1733691100

Rasouly A, Ron EZ. 2009. Interplay between the heat shock response and translation in Escherichia coli. Res Microbiol 160: 288-296. doi:10.1016/j.resmic.2009.03.007

Romilly C, Deindl S, Wagner EGH. 2019. The ribosomal protein S1dependent standby site in tisB mRNA consists of a single-stranded region and a 5' structure element. Proc Natl Acad Sci 116: 1590115906. doi:10.1073/pnas. 1904309116

Sacerdot C, Caillet J, Graffe M, Eyermann F, Ehresmann B, Ehresmann C, Springer M, Romby P. 1998. The Escherichia coli threonyl-tRNA synthetase gene contains a split ribosomal binding site interrupted by a hairpin structure that is essential for autoregulation. Mol Microbiol 29: 1077-1090. doi:10.1046/j.1365-2958 .1998.00995.x
Schultzaberger RK, Chen Z, Lewis KA, Schneider TD. 2007. Anatomy of Escherichia coli $\sigma^{70}$ promoters. Nucleic Acids Res 35: 771-788. doi:10.1093/nar/gkl956

Shasmal M, Chakraborty B, Sengupta J. 2010. Intrinsic molecular properties of the protein-protein bridge facilitate ratchet-like motion of the ribosome. Biochem Biophys Res Commun 399: 192197. doi:10.1016/j.bbrc.2010.07.053

Sigdel TK, Easton JA, Crowder MW. 2006. Transcriptional response of Escherichia coli to TPEN. J Bacteriol 188: 6709-6713. doi:10 .1128/JB.00680-06

Smith C, Heyne S, Richter AS, Will S, Backofen R. 2010. Freiburg RNA Tools: a web server integrating IntaRNA, ExpaRNA and LocARNA. Nucleic Acids Res 38: W373-W377. doi:10.1093/nar/gkq316

Sterk M, Romilly C, Wagner EGH. 2018. Unstructured 5'-tails act through ribosome standby to override inhibitory structure at ribosome binding sites. Nucleic Acids Res 46: 4188-4199. doi:10 $.1093 /$ nar/gky073

Tchufistova LS, Komarova AV, Boni IV. 2003. A key role for the mRNA leader structure in translational control of ribosomal protein S1 synthesis in $\gamma$-proteobacteria. Nucleic Acids Res 31: 6996-7002. doi:10.1093/nar/gkg883

Thomason MK, Bischler T, Eisenbart SK, Förstner KU, Zhang A, Herbig A, Nieselt K, Sharma CM, Storz G. 2015. Global transcriptional start site mapping using differential RNA sequencing reveals novel antisense RNAs in Escherichia coli. J Bacteriol 197: 18-28. doi:10.1128/JB.02096-14

Ueta M, Wada C, Bessho Y, Maeda M, Wada A. 2017. Ribosomal protein L31 in Escherichia coli contributes to ribosome subunit association and translation, whereas short L31 cleaved by protease 7 reduces both activities. Genes Cells 22: 452-471. doi:10.1111/ gtc. 12488

Wade JT, Castro Roa D, Grainger DC, Hurd D, Busby SJ, Struhl K, Nudler E. 2006. Extensive functional overlap between $\sigma$ factors in Escherichia coli. Nat Struct Mol Biol 13: 806-814. doi:10 $.1038 / \mathrm{nsmb} 1130$

Zengel JM, Lindahl L. 1994. Diverse mechanisms for regulating ribosomal protein synthesis in Escherichia coli. Prog Nucleic Acids Res Mol Biol 47: 331-370. doi:10.1016/S0079-6603(08)60256-1

Zuker M. 2003. Mfold web server for nucleic acid folding and hybridization prediction. Nucleic Acids Res 31: 3406-3415. doi:10.1093/ nar/gkg595 

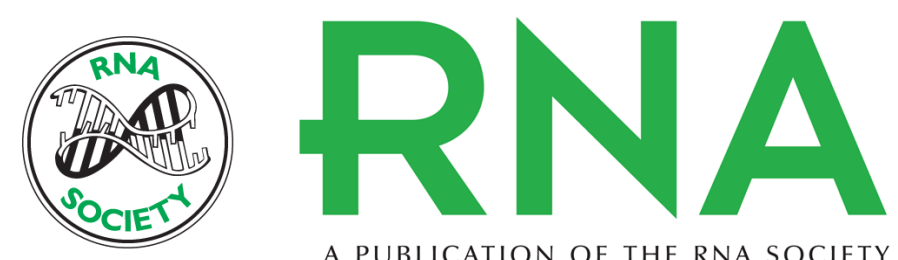

A PUBLICATION OF THE RNA SOCIETY

\section{Autogenous regulation in vivo of the rpmE gene encoding ribosomal protein L31 (bL31), a key component of the protein-protein intersubunit bridge B1b}

Leonid V. Aseev, Ludmila S. Koledinskaya and Irina V. Boni

RNA 2020 26: 814-826 originally published online March 24, 2020

Access the most recent version at doi:10.1261/rna.074237.119

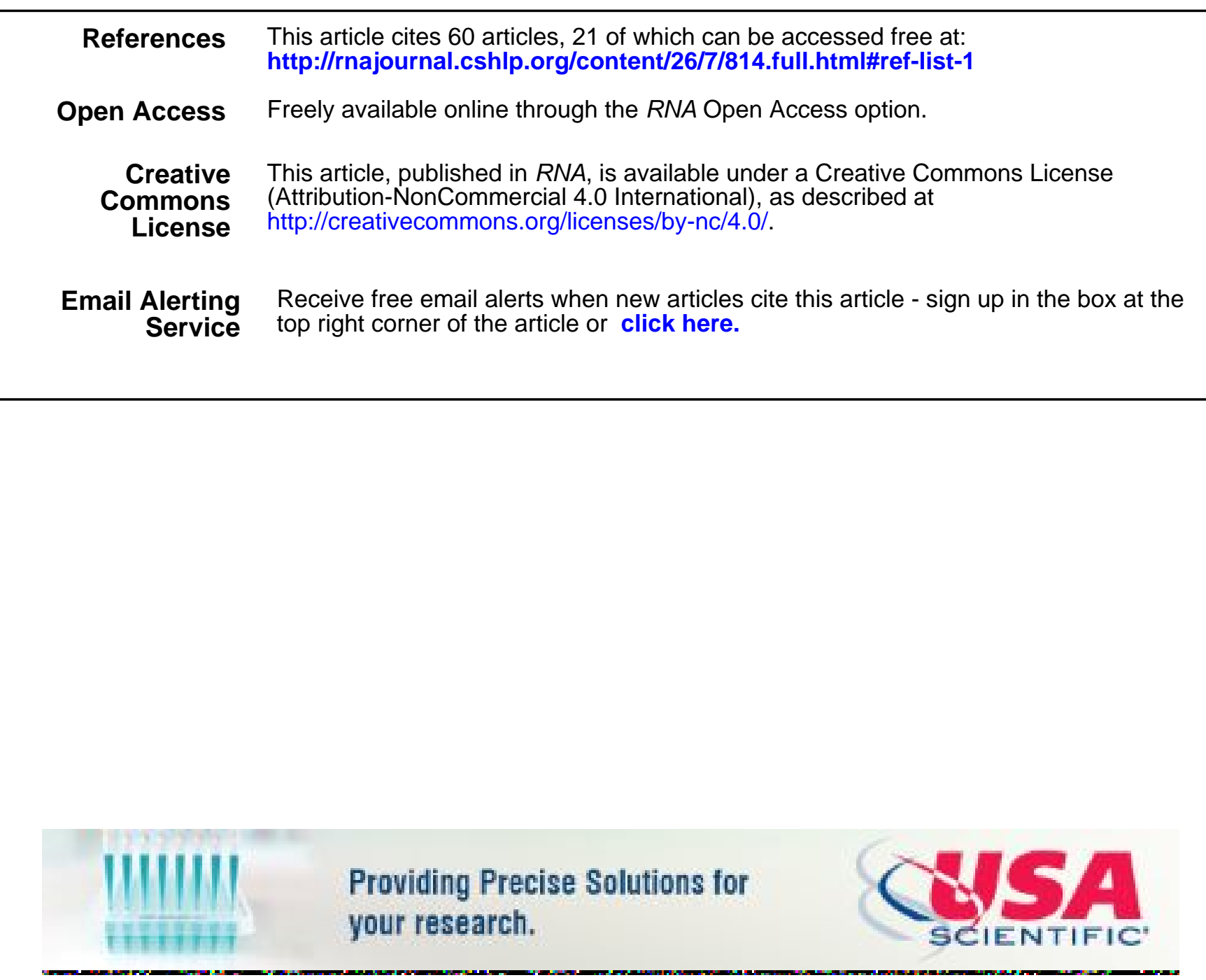

To subscribe to RNA go to:

http://rnajournal.cshlp.org/subscriptions

(C) 2020 Aseev et al.; Published by Cold Spring Harbor Laboratory Press for the RNA Society 